\title{
Molecular Determinants of Prognosis in Hepatocellular Carcinoma
}

\author{
Jean-Charles Nault ${ }^{1,2,3}$ \\ ${ }^{1}$ Inserm, UMR-1162, Génomique fonctionnelle des Tumeurs solides, IUH, Paris, France; ${ }^{2}$ Université Paris Descartes, Labex \\ Immuno-Oncology, Sorbonne Paris Cité, Faculté de Médecine, Paris, France; ${ }^{3}$ Service d'Hépatologie, Hôpital Jean Verdier, AP-HP, \\ Bondy, and Université Paris 13, Bobigny, France
}

\begin{abstract}
Hepatocellular carcinoma is one of the leading causes of death by cancer worldwide. Prognosis of hepatocellular carcinoma is determined by characteristics of the tumor and the surrounding cirrhotic liver. Several molecular signatures reflecting tumor biology and derived from tumor analyses predict early tumor recurrence and survival. In contrast, molecular signatures from cirrhotic non-tumor samples are enriched in immunity/inflammation related genes and could predict late tumor recurrence. Moreover, combination of clinical, pathological, and molecular features may refine prognosis prediction in these patients. Finally, molecular signatures from both tumor and non-tumor tissues will be helpful in the future to guide treatments in different clinical settings.

(c) 2014 The Second Affiliated Hospital of Chongqing Medical University. Published by XIA \& HE Publishing Ltd. All rights reserved.
\end{abstract}

\section{Introduction}

Hepatocellular carcinoma (HCC) is one of the most frequent primitive liver malignancies, followed by cholangiocarcinoma, and involves malignant proliferation of hepatocytes. ${ }^{1,2}$ The main etiologies of HCC are chronic hepatitis B (HBV) and C infection (HCV), alcoholic liver disease, metabolic syndrome (non-alcoholic steatohepatitis (NASH)), and hemochromatosis. Worldwide, the incidence of HCC is heterogeneous and

Keywords: Hepatocellular carcinoma; Molecular signature; Prognosis; Microarray; Cirrhosis; Liver resection.

Abbreviations: AASLD, American Association for the Study of Liver Diseases AFP, alpha-fetoprotein; ARID1A, AT-rich interactive domain-containing protein 1A; ARID2, AT-rich interactive domain-containing protein 2; BCLC, Barcelona Clinic Liver Cancer; CK7, cytokeratin 7; CK19, cytokeratin 19; CTNNB1, Catenin (Cadherin-Associated Protein), Beta 1; EASL, European Association for the Study of the Liver; EPCAM, Epithelial cell adhesion molecule; FFPE, formalin-fixed, paraffin-embedded; HBV, hepatitis B; HCC, Hepatocellular carcinoma; HCV, hepatitis C; HER2, human epidermal growth factor receptor 2; IDH1, isocitrate dehydrogenase 1; KEAP1, Kelch-like ECH-associated protein 1; MLL4, Myeloid/ lymphoid or mixed-lineage leukemia $4 ; \mathrm{NASH}$, non-alcoholic steatohepatitis; NFE2L2, Nuclear factor (erythroid-derived 2)-like 2; NFKB, nuclear factor-kappa B; RPS6KA3, Ribosomal protein S6 kinase, 90kDa, polypeptide 3; TERT, telomerase reverse transcriptase; TP53, Tumor protein p53.

Received: 10 December 2013; Revised: 25 January 2014; Accepted: 26 January 2014

DOI of original article: 10.14218/JCTH.2013.00029.

Correspondence to: Jean-Charles Nault, INSERM UMR-674 Université Paris Descartes, 27 rue Juliette Dodu, Paris 75010, France. Tel: +33-1-53-72-51-94, Fax: +33-1-53-72-51-92, Email: naultjc@gmail.com follows the geographic distribution of the underlying etiology. ${ }^{3}$ HBV infection is responsible for most cases in Asian countries, while HCV, alcohol consumption, and NASH are the causes of most cases in western countries. In western countries, HCC develops primarily following cirrhosis. In Asia, HCC may occur in non-cirrhotic liver due to the direct carcinogenic effect of $\mathrm{HBV}^{3}$ European Association for the Study of the Liver (EASL) and American Association for the Study of Liver Diseases (AASLD) recommend the Barcelona Clinic Liver Cancer (BCLC) classification for stratifying HCC patients and guiding treatment. ${ }^{4}$ The main curative treatments are liver resection, radiofrequency, and liver transplantation. ${ }^{1}$ Liver resection and radiofrequency ablation are impaired by a high rate of tumor recurrence. ${ }^{5}$ In contrast, liver transplantation enables long-term survival with a low rate of recurrence but is possible only for early HCC and is limited by organ shortage. ${ }^{4}$ Most cases, however, present at advanced stages and can only be treated by transarterial chemo-embolization or sorafenib., ${ }^{6,7}$ A precise prognosis for these patients is of utmost importance in guiding clinical decisions and stratifying treatments.

\section{General considerations concerning prognosis assessment in patients with HCC}

The prognosis in HCC is related to features of both tumoral and non-tumoral liver. $^{8}$ Moreover, clinical and molecular studies revealed two patterns of tumor recurrence closely related to biological and clinical characteristics: early recurrence during the 2-3 years following surgery and late recurrence occurring 2-3 years after surgery. ${ }^{9}$ Early recurrence is driven by tumor features and is related to intrahepatic metastasis of the original tumor. In contrast, late recurrence is characterized by de novo carcinogenesis of cirrhosis and is related to cirrhotic features. ${ }^{8-10}$ This dichotomy is clear-cut with curative treatments such as radiofrequency ablation and liver resection, where the cirrhotic liver is still present after treatment. In liver transplantation, the diseased liver is removed and tumor recurrence is conditioned only by tumor features. At advanced stages, the patient's prognosis is mainly linked to HCC progression and tumor features. These observations have several potential implications for therapy. Secondary chemoprevention to avoid the occurrence of new HCC of cirrhosis can be useful in patients treated by resection or percutaneous ablation, but is useless in patients treated by liver transplantation or with advanced HCC. ${ }^{11}$ In contrast, analysis of biological features of $\mathrm{HCC}$ is necessary to personalize target therapy at each stage 
of the disease. ${ }^{8}$ Cirrhotic patients, however, may also die from liver failure due to cirrhosis independently of tumor recurrence. All three factors (tumor recurrence due to the initial tumor, de novo carcinogenesis, and liver failure not related to tumor progression) must be taken into account in order to accurately predict patient prognosis.

\section{Pitfalls in the prognostic molecular signature}

Since Golub et al. in 1999 first molecularly classified tumors using microarray, ${ }^{12}$ several groups have reported molecular classification and prognostic molecular signatures in HCC. ${ }^{13}$ However, the potential application to clinical practice of these different signatures should be viewed with caution. Several guidelines (including the REMARK statement, the PROGRESS statement, and EASL guidelines for HCC) have paved the way for the development of prognostic biomarkers that are robust, reproducible, and have potential translation into clinical practice: ${ }^{4,14,15}$

- Firstly, in order to avoid overestimation of the value of biomarkers in the training set, identification of biomarkers should be performed using a training cohort set followed by a validation set (Fig. 1). ${ }^{14,15}$

- Secondly, the biomarkers should retain their prognostic value when compared to classical clinical and pathological features (serum alpha-fetoprotein (AFP), Barcelona Clinic Liver Cancer (BCLC) staging, severity of cirrhosis, presence of portal hypertension, size and number of tumors, microvascular invasion, satellite nodules, etc. ). ${ }^{9,16}$ Most of the oldest studies on HCC prognostic molecular signatures did not follow these recommendations.

- Finally, the different guidelines concerning prognostic biomarkers should be validated externally by other
Nault JC.: Molecular prognosis of hepatocellular carcinoma

groups, with the aim of testing the robustness and reproducibility of the molecular signature (Fig. 1). ${ }^{4}$ This is a key point, since HCC develops at different stages of liver disease and is due to various etiologies worldwide. This heterogeneity should be taken into account for future use of prognostic biomarkers in clinical practice.

\section{Prognostic molecular signatures of tumors}

More than 20 different prognostic molecular signatures from tumor tissue have been currently published. ${ }^{17,18}$ Here, we detail some of these molecular signatures:

\section{Proliferative subclasses}

One seminal study was published by Snorri Thorgeisson's group in 2004. ${ }^{19}$ Using microarray, they distinguished two major groups, named A and B. HCC patients classified into group A had a worse prognosis. This subgroup was also called the "proliferative" class because it was characterized by deregulation of cell cycle and proliferation genes. ${ }^{19}$

\section{HCC with stem cell features}

The same group reported that HCC harboring stem cell features ("hepatoblast-like") had a high risk of recurrence and death. ${ }^{20}$ These stem cell characteristics were also detected using immunohistochemical markers like cytokeratin 7 (CK7), cytokeratin 19 (CK19), and epithelial cell adhesion molecule (EPCAM). ${ }^{21,22}$ However, three recent studies did not reproduce the prognostic value of stem cell features in early $\mathrm{HCC}^{23-25}$ This could be explained by the lower prevalence of stem cell features in early HCC

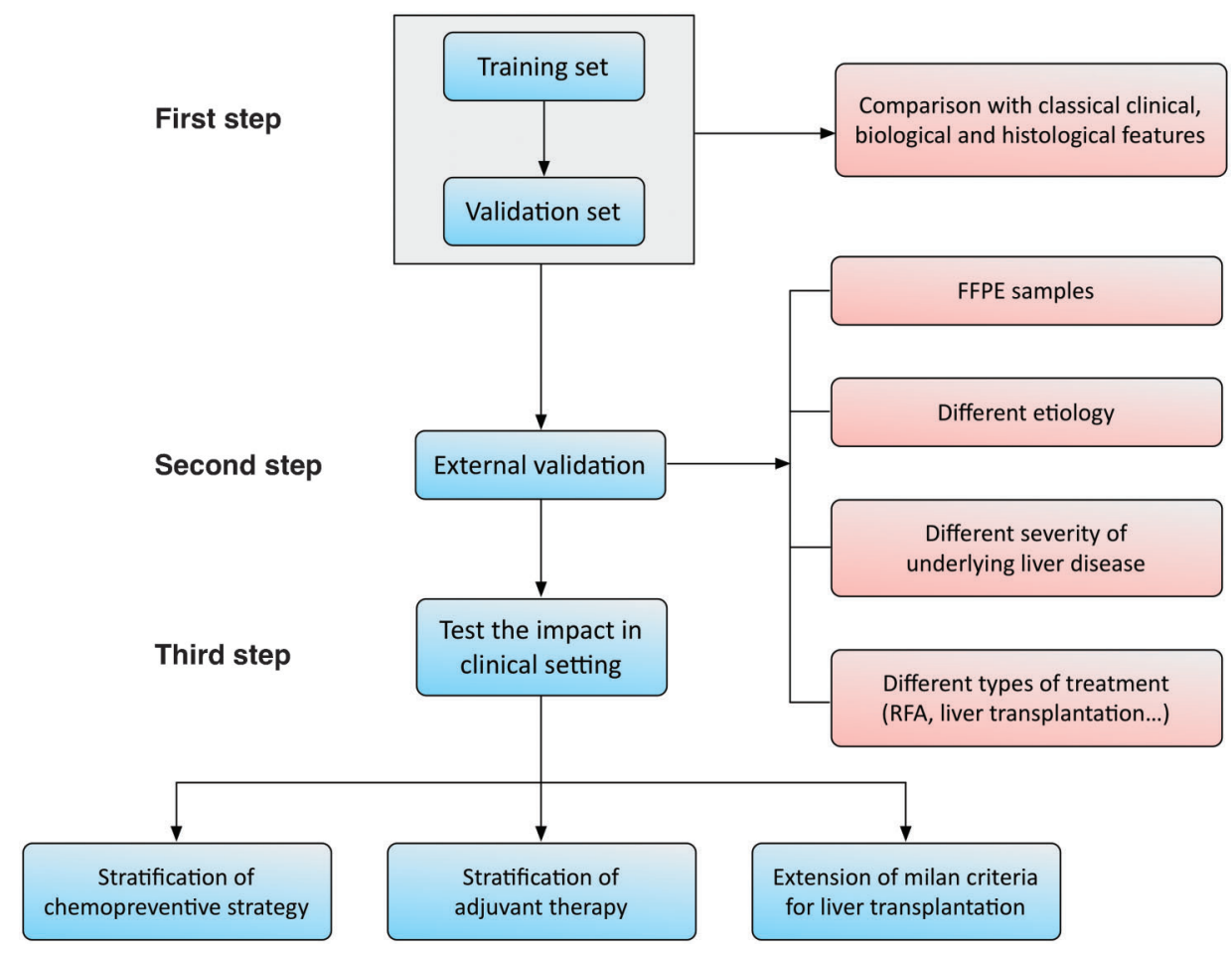

Fig. 1. Different steps for biomarker prognostic studies. RFA: radiofrequency ablation, FFPE: formalin-fixed, paraffin-embedded 
Nault JC.: Molecular prognosis of hepatocellular carcinoma

\section{Tumor biology}

\begin{tabular}{|c|}
\hline Serum AFP \\
Numbers/Size of HCC \\
Microvascular invasion \\
Differentiation \\
Satellite nodules \\
Anatomic resection CK19 \\
immunostaining
\end{tabular}

\begin{tabular}{c} 
5-gene score ${ }^{24}$ \\
Proliferative \\
subclass/Cluster $\mathrm{A}^{19}$ \\
G3 subgroup ${ }^{26}$ \\
Hepatoblast signature ${ }^{20}$ \\
Metastasis signature \\
Mir26 \\
\hline
\end{tabular}

\section{De novo carcinogenesis}

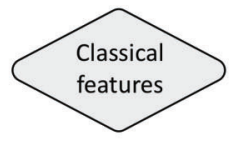

Portal hypertension

Child Pugh

Gender

Cytolysis

Control of viral infection

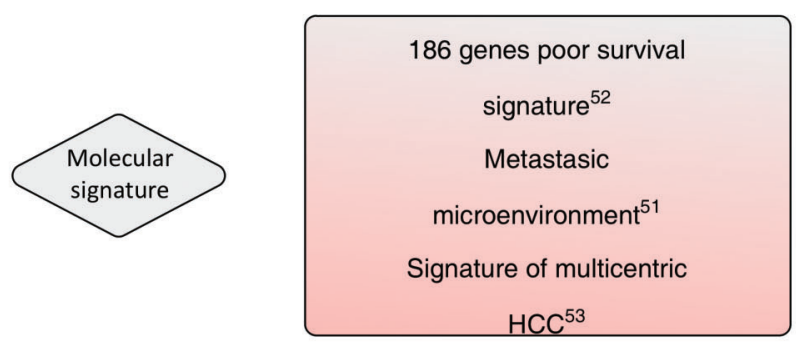

$\mathrm{HCC}^{53}$
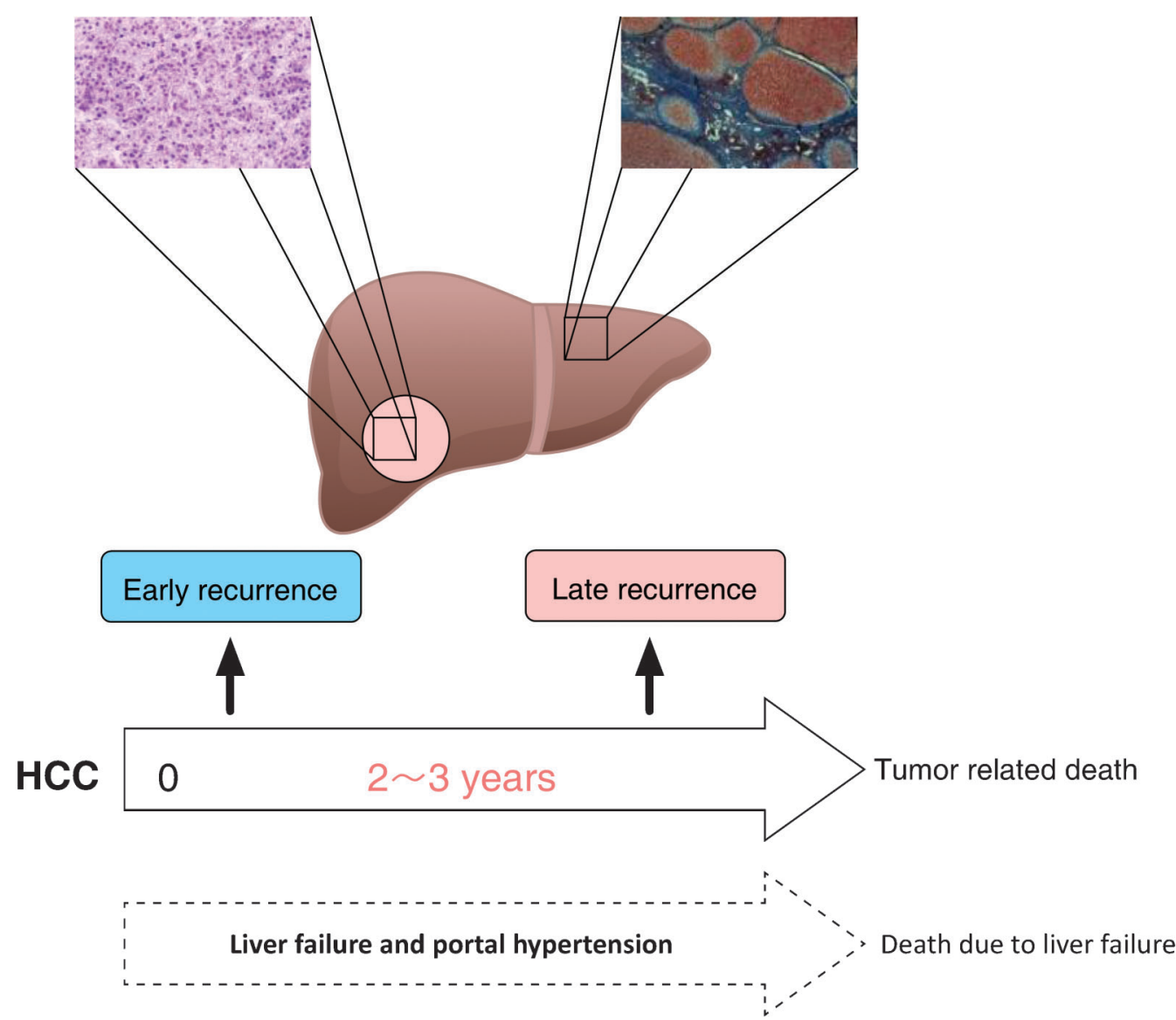

Fig. 2. Molecular determinants of prognosis after HCC resection on cirrhosis. Some examples of molecular signatures related to tumor biology and to features of cirrhotic non tumor liver (carcinogenic field effect) are shown. 
developing in HCV-related or alcohol-related cirrhosis compared to the higher frequency in advanced HCC- or HBVrelated $\mathrm{HCC}$.

\section{G1-G6 classification}

We reported molecular classification of tumors into 6 subgroups (G1-G6) based on closely related clinical and genetic features. ${ }^{26} \mathrm{G} 1-\mathrm{G} 3$ subgroups were characterized by chromosomal instability and G4-G6 subgroups by chromosomal stability. The G1 subgroup was characterized by reactivation of fetal genes and the presence of stem cell markers in young women with high AFP levels. The G1-G2 subgroup was associated with HBV chronic infection. The G3 subgroup was associated with poor prognosis. ${ }^{23,26}$ These tumors have inactivating mutations of TP53 and inactivation of CDKN2A by promoter methylation. ${ }^{27}$ This suggests that co-occurrence of inactivation of $P 53$ and the retinoblastoma pathway could be a reason for poor prognosis of these tumors. In addition, G3 HCC harbored dysregulation of genes coding proteins of the nuclear pore and cell cycle. G5-G6 subgroups were strongly associated with activating mutations of B-catenin (coded by CTNNB1). Recently, a study compiled eighteen prognostic molecular signatures from tumors to assess their validity in a series of $287 \mathrm{HCC}$ developing in cirrhosis and treated by liver resection. ${ }^{19,20,23,26,28-32}$ Strikingly, among 18 different prognostic molecular signatures, HCC classified by our G3 subgroup was the most accurate biomarker for predicting early tumor recurrence.

\section{The 5-gene score}

Recently, we developed a 5-gene score in order to accurately predict the prognosis for HCC treated by liver resection. ${ }^{24}$ This simple molecular signature was derived from analysis of tumor tissues and dichotomized patients into good and poor prognosis groups. The 5-gene score was able to accurately predict early recurrence and overall survival of patients with HCC treated by liver resection in France, independently of clinical and pathological features. The 5-gene score also more accurately predicted risk of death compared to the G3 signature. ${ }^{24}$ In addition, this molecular signature was externally validated in one series of 213 cirrhotic patients with early HCC from western countries and one series of 221 patients with HBV-related HCC from Asia. In addition to this reproducibility, regardless of underlying liver disease and etiology, the 5-gene score was validated in formalin-fixed, paraffin-embedded (FFPE) samples. ${ }^{24}$ This assured its robustness for potential use in the future.

\section{Mir-26 and response to interferon therapy}

Moreover, some prognostic signatures were derived from micro-RNA analysis of HCC. ${ }^{33,34}$ Patients with low mir-26 expression in the tumor had shorter overall survival in three cohorts of 455 patients with HCC treated by liver resection in Asia. ${ }^{35}$ In addition, low mir-26 expression predicted a satisfactory response to adjuvant interferon therapy. That study provided the link between prognostic value (predicting prognosis independently of treatment) and predictive value (predicting response to a specific treatment) of a molecular test. ${ }^{36}$ However, that study focused on Asian patients infected by hepatitis $B$ and needs to be reproduced in western patients with alcohol- or hepatitis-C-related cirrhosis.

\section{Prognostic values of somatic genetic alterations}

Finally, like other cancers, HCC is a disease of the genome and is characterized by an accumulation of genetic alterations in a somatic manner. In several types of cancer, genetic alterations have been linked with prognosis. For example, isocitrate dehydrogenase 1 (IDH1) mutation is associated with good prognosis in glioblastoma and human epidermal growth factor receptor 2 (HER2) amplification with poor prognosis in breast cancer. ${ }^{37-39}$ In HCC, both candidate gene studies and, more recently, next-generation studies have refined our understanding of genetic alterations of HCC. The most frequent genetic alterations are telomerase reverse transcriptase (TERT) promoter mutations (59\%), Tumor protein p53 (TP53) mutations (20-40\%), and Catenin (Cadherin-Associated Protein), Beta 1 (CTNNB1) mutations (20-30\%)..$^{40-42}$ TERT promoter mutations were not associated with prognosis in a series of western patients treated by liver resection, but additional studies are required in HCC having different etiologies and at different stages. ${ }^{42}$ There is also a substantial discrepancy in the prognostic value of CTNNB1 (coding for B-catenin) mutations, ranging from good to poor prognosis. ${ }^{24,43-45}$ Finally, TP53 mutations were associated with poor prognosis after liver resection of HBVrelated HCC from Asia. ${ }^{46-48}$ HCC with TP53 mutations also harbored stem cell markers. In contrast, in a series of western patients with HCC treated by liver resection, TP53 mutations were not associated with tumor recurrence or overall survival. ${ }^{24}$ This could indicate that prognostic biomarkers vary according to etiology and underlying liver disease. Other recurrent genetic alterations have been described in HCC, including ARID1A (AT-rich interactive domain-containing protein 1A), ARID2 (AT-rich interactive domain-containing protein 2), NFE2L2 (Nuclear factor (erythroid-derived 2)-like 2), RPS6KA3 (Ribosomal protein S6 kinase, 90kDa, polypeptide 3), MLL4 (Myeloid/lymphoid or mixed-lineage leukemia 4), and KEAP1 (Kelch-like ECHassociated protein 1) mutations, but their prognostic value alone and in combination remains unknown. ${ }^{41,49,50}$

\section{Prognostic molecular signatures from non-tumoral cirrhotic tissue}

Biological features of the surrounding non-tumoral liver is strongly related to so-called "de novo carcinogenesis", with both occurrence of HCC in cirrhotic patients without HCC at baseline and late recurrence after resection of HCC. ${ }^{10,51-53}$ The first study that addressed this question at the molecular level was conducted by the team of XW Wang. ${ }^{51}$ They identified a signature of non-tumoral liver that predicted overall survival. The gene included in this signature was enriched in immune/inflammatory genes. ${ }^{51}$ Moreover, another study identified a 186-"poor survival"-signature derived from analysis of cirrhotic tissue in patients treated by liver resection for $\mathrm{HCC}{ }^{52}$ This signature predicted both overall survival and late recurrence but not early recurrence. That study provided molecular proof of principle that nontumoral tissue in cirrhotic patients with HCC was strongly linked to de novo carcinogenesis and late recurrence. ${ }^{52}$ In addition, that paper showed that FFPE tissues could be used to assess molecular patterns and paved the way for potential use of molecular signatures in clinical practice. Indeed, FFPE samples are more widely available than frozen ones. Strikingly, genes with the "poor survival" signature belonged 
to the pathway of interferon signaling, tumor necrosis factor signaling, and the nuclear factor-kappa B (NFKB) pathway. ${ }^{52}$ These two studies converged and pinpointed immunity and inflammation as key pathogenic events in de novo liver carcinogenesis. ${ }^{10}$ Recently, these 186 -gene signatures were tested in FFPE samples from biopsies of cirrhotic patients without HCC. ${ }^{54}$ They were able to predict death, HCC occurrence, and progression to advanced cirrhosis. This suggests that molecular signatures from non-tumoral liver reflect the severity of cirrhosis and could predict both de novo carcinogenesis and death from liver failure. ${ }^{8,10}$

\section{A combination of molecular signatures and clinical and pathological features for refining prognosis assessment}

Since the molecular signature from tumoral and non-tumoral tissues captures different biological signals and is able to predict different clinical events, we hypothesized that the association of these two predictive features would refine the assessment of prognosis. Along this line, we showed that the association of the 5-gene score from the tumor and the 186-gene "poor survival" signature from the non-tumoral tissue would more accurately predict the prognosis than each signature alone. ${ }^{24,52}$ Interestingly, patients classified into the poor prognosis group by both the 5-gene score and the 186poor survival signature had the worst prognoses. ${ }^{24}$ Moreover, most studies had compared clinical and pathological features with molecular features. ${ }^{23}$ However, the prognostic value of clinical, pathological, and molecular features could be combined to refine the assessment of prognosis. We mixed clinical features (the BCLC classification), pathological features (presence or absence of microvascular invasion), and molecular scores (the 5-genes score) into a nomogram that provided individual risk of death for each patient. ${ }^{24}$

\section{Conclusions}

Huge advances have been made in the field of molecular classification and molecular assessment of the prognosis of HCC. At present, no molecular signature has been validated in the setting of radiofrequency ablation, liver transplantation, or advanced HCC treated by chemo-embolization or sorafenib. ${ }^{55}$ Likewise, the validation of different molecular signatures in tumor biopsies will be required for use in clinical practice. ${ }^{4,56}$ Consequently, the most important step is to translate this molecular tool into clinical practice in an easy, robust manner (Fig. 1). Importantly, this new tool should be tested to guide clinical decisions in various settings. ${ }^{57}$ In cirrhotic patients, identification of patients at high risk for HCC could be useful for testing chemoprophylaxis or in intensive screening strategy. ${ }^{11,58}$ In patients treated by curative methods such as liver resection and radiofrequency ablation, molecular signatures from tumor tissues could stratify patients at high risk of recurrence so as to adapt adjuvant treatment. $8,59,60$ In the same clinical setting, assessment of the carcinogenic potential of cirrhotic tissues by molecular tools would be useful for selecting guided secondary chemoprophylaxis. ${ }^{10}$ Finally, the molecular signature could also be used to select patients who will benefit from liver transplantation. ${ }^{61}$ Patients with HCC falling within the Milan criteria and classified into the poor prognosis group should receive aggressive neoadjuvant/waiting treatment or possibly be excluded from the waiting list if they progress under treatment. In contrast, patients falling outside the
Milan criteria and classified within the good prognosis group by molecular tools could be transplanted. ${ }^{62,63}$ However, this type of strategy should be tested prospectively in order to validate the concept of personalized medicine in the field of liver cancer.

\section{Conflict of interest}

None

\section{Author contributions}

Conceiving and writing the manuscript (JCN).

References

[1] El-Serag HB. Hepatocellular carcinoma. N Engl J Med 2011;365:1118-1127.

[2] Farazi PA, DePinho RA. Hepatocellular carcinoma pathogenesis: from genes to environment. Nat Rev Cancer 2006;6:674-687.

[3] Forner A, Llovet JM, Bruix J. Hepatocellular carcinoma. Lancet 2012;379: 1245-1255.

[4] European Association For The Study Of The Liver. EASL-EORTC clinical practice guidelines: management of hepatocellular carcinoma. J Hepatol 2012; 56:908-943.

[5] Beaugrand M, N'Kontchou G, Seror O, Ganne N, Trinchet JC. Local/regional and systemic treatments of hepatocellular carcinoma. Semin Liver Dis 2005; 25:201-211.

[6] Llovet JM, Ricci S, Mazzaferro V, Hilgard P, Gane E, Blanc JF, et al. Sorafenib in advanced hepatocellular carcinoma. N Engl J Med 2008;359:378-390.

[7] Llovet JM, Bruix J. Systematic review of randomized trials for unresectable hepatocellular carcinoma: Chemoembolization improves survival. Hepatology 2003;37:429-442.

[8] Villanueva A, Hoshida Y, Toffanin S, Lachenmayer A, Alsinet C, Savic R, et al. New strategies in hepatocellular carcinoma: genomic prognostic markers. Clin Cancer Res 2010;16:4688-4694.

[9] Imamura H, Matsuyama $Y$, Tanaka E, Ohkubo T, Hasegawa K, Miyagawa S, et al. Risk factors contributing to early and late phase intrahepatic recurrence of hepatocellular carcinoma after hepatectomy. J Hepatol 2003;38:200-207.

[10] Hernandez-Gea V, Toffanin S, Friedman SL, Llovet JM. Role of the microenvironment in the pathogenesis and treatment of hepatocellular carcinoma. Gastroenterology 2013;144:512-527.

[11] Singh S, Singh PP, Roberts LR, Sanchez W. Chemopreventive strategies in hepatocellular carcinoma. Nat Rev Gastroenterol Hepatol 2014:11:45-54.

[12] Golub TR, Slonim DK, Tamayo P, Huard C, Gaasenbeek M, Mesirov JP, et al. Molecular classification of cancer: class discovery and class prediction by gene expression monitoring. Science 1999;286:531-537.

[13] Reis-Filho JS, Pusztai L. Gene expression profiling in breast cancer: classification, prognostication, and prediction. Lancet 2011;378:18121823.

[14] McShane LM, Altman DG, Sauerbrei W, Taube SE, Gion M, Clark GM Reporting recommendations for tumor marker prognostic studies (REMARK). J Natl Cancer Inst 2005;97:1180-1184.

[15] Riley RD, Hayden JA, Steyerberg EW, Moons KG, Abrams K, Kyzas PA, et al. Prognosis Research Strategy (PROGRESS) 2: prognostic factor research. PLoS Med 2013;10:e1001380.

[16] Ishizawa T, Hasegawa K, Aoki T, Takahashi M, Inoue Y, Sano K, et al. Neither multiple tumors nor portal hypertension are surgical contraindications for hepatocellular carcinoma. Gastroenterology 2008;134:1908-1916.

[17] Hoshida Y, Toffanin S, Lachenmayer A, Villanueva A, Minguez B, Llovet JM. Molecular classification and novel targets in hepatocellular carcinoma: recent advancements. Semin Liver Dis 2010;30:35-51.

[18] Lee JS, Thorgeirsson SS. Genome-scale profiling of gene expression in hepatocellular carcinoma: classification, survival prediction, and identification of therapeutic targets. Gastroenterology 2004;127:S51-S55.

[19] Lee JS, Chu IS, Heo J, Calvisi DF, Sun Z, Roskams T, et al. Classification and prediction of survival in hepatocellular carcinoma by gene expression profiling. Hepatology 2004;40:667-676.

[20] Lee JS, Heo J, Libbrecht L, Chu IS, Kaposi-Novak P, Calvisi DF, et al. A nove prognostic subtype of human hepatocellular carcinoma derived from hepatic progenitor cells. Nat Med 2006;12:410-416.

[21] Yamashita T, Ji J, Budhu A, Forgues M, Yang W, Wang HY, et al. EpCAMpositive hepatocellular carcinoma cells are tumor-initiating cells with stem/ progenitor cell features. Gastroenterology 2009;136:1012-1024.

[22] Durnez A, Verslype C, Nevens F, Fevery J, Aerts R, Pirenne J, et al. The clinicopathological and prognostic relevance of cytokeratin 7 and 19 
expression in hepatocellular carcinoma. A possible progenitor cell origin. Histopathology 2006;49:138-151.

[23] Villanueva A, Hoshida Y, Battiston C, Tovar V, Sia D, Alsinet C, et al. Combining clinical, pathology, and gene expression data to predict recurrence of hepatocellular carcinoma. Gastroenterology 2011;140:1501-151.

[24] Nault JC, De Reynies A, Villanueva A, Calderaro J, Rebouissou S, Couchy G, et al. A hepatocellular carcinoma 5-gene score associated with survival of patients after liver resection. Gastroenterology 2013;145:176-187.

[25] Ziol M, Sutton A, Calderaro J, Barget N, Aout M, Leroy V, et al. ESM-1 expression in stromal cells is predictive of recurrence after radiofrequency ablation in early hepatocellular carcinoma. J Hepatol 2013;59:1264-1270.

[26] Boyault S, Rickman DS, de Reynies A, Balabaud C, Rebouissou S, Jeannot E, et al. Transcriptome classification of $\mathrm{HCC}$ is related to gene alterations and to new therapeutic targets. Hepatology 2007;45:42-52.

[27] Munro S, Carr SM, La Thangue NB. Diversity within the pRb pathway: is there a code of conduct? Oncogene 2012;31:4343-4352.

[28] Roessler S, Jia HL, Budhu A, Forgues M, Ye QH, Lee JS, et al. A unique metastasis gene signature enables prediction of tumor relapse in early-stage hepatocellular carcinoma patients. Cancer Res 2010;70:10202-10212.

[29] Kaposi-Novak P, Lee JS, Gomez-Quiroz L, Coulouarn C, Factor VM, Thorgeirsson SS. Met-regulated expression signature defines a subset of human hepatocellular carcinomas with poor prognosis and aggressive phenotype. J Clin Invest 2006;116:1582-1595.

[30] Villanueva A, Chiang DY, Newell P, Peix ], Thung S, Alsinet C, et al. Pivotal role of mTOR signaling in hepatocellular carcinoma. Gastroenterology 2008;135: 1972-1983.

[31] Woo HG, Park ES, Cheon JH, Kim JH, Lee JS, Park BJ, et al. Gene expressionbased recurrence prediction of hepatitis $\mathrm{B}$ virus-related human hepatocellular carcinoma. Clin Cancer Res 2008;14:2056-2064.

[32] Woo HG, Lee JH, Yoon JH, Kim CY, Lee HS, Jang JJ, et al. Identification of a cholangiocarcinoma-like gene expression trait in hepatocellular carcinoma. Cancer Res 2010;70:3034-3041.

[33] Wang XW, Heegaard NH, Orum H. MicroRNAs in liver disease. Gastroenterology 2012;142:1431-1443.

[34] Budhu A, Jia HL, Forgues M, Liu CG, Goldstein D, Lam A, et al. Identification of metastasis-related microRNAs in hepatocellular carcinoma. Hepatology 2008;47:897-907

[35] Ji J, Shi J, Budhu A, Yu Z, Forgues M, Roessler S, et al. MicroRNA expression, survival, and response to interferon in liver cancer. N Engl J Med 2009;361: 1437-1447.

[36] Ji J, Yu L, Yu Z, Forgues M, Uenishi T, Kubo S, et al. Development of a miR-26 companion diagnostic test for adjuvant interferon-alpha therapy in hepatocellular carcinoma. Int J Biol Sci 2013;9:303-312.

[37] Houillier C, Wang X, Kaloshi G, Mokhtari K, Guillevin R, Laffaire J, et al. IDH1 or IDH2 mutations predict longer survival and response to temozolomide in low-grade gliomas. Neurology 2010;75:1560-1566.

[38] Ross JS, Fletcher JA. The HER-2/neu oncogene in breast cancer: prognostic factor, predictive factor, and target for therapy. Oncologist 1998;3:237-252.

[39] McDermott U, Downing JR, Stratton MR. Genomics and the continuum of cancer care. N Engl J Med 2011;364:340-350.

[40] Nault JC, Zucman-Rossi J. Genetics of hepatobiliary carcinogenesis. Semin Liver Dis 2011;31:173-187.

[41] Guichard C, Amaddeo G, Imbeaud S, Ladeiro Y, Pelletier L, Maad IB, et al. Integrated analysis of somatic mutations and focal copy-number changes identifies key genes and pathways in hepatocellular carcinoma. Nat Genet 2012;44:694-698.

[42] Nault JC, Mallet M, Pilati C, Calderaro J, Bioulac Sage P, Laurent C, et al. High frequency of telomerase reverse-transcriptase promoter somatic mutations in hepatocellular carcinoma and preneoplastic lesions. Nat Commun 2013;4: 2218.

[43] Hsu HC, Jeng YM, Mao TL, Chu JS, Lai PL, Peng SY. $\beta$-catenin mutations are associated with a subset of low-stage hepatocellular carcinoma negative for hepatitis B virus and with favorable prognosis. Am J Pathol 2000;157: $763-770$.
[44] Mao TL, Chu JS, Jeng YM, Lai PL, Hsu HC. Expression of mutant nuclear $\beta$-catenin correlates with non-invasive hepatocellular carcinoma, absence of portal vein spread, and good prognosis. J Pathol 2001;193:95-101.

[45] Inagawa S, Itabashi M, Adachi S, Kawamoto T, Hori M, Shimazaki J, et al. Expression and prognostic roles of $\beta$-catenin in hepatocellular carcinoma: correlation with tumor progression and postoperative survival. Clin Cancer Res 2002;8:450-456.

[46] Woo HG, Wang XW, Budhu A, Kim YH, Kwon SM, Tang ZY, et al. Association of TP53 mutations with stem cell-like gene expression and survival of patients with hepatocellular carcinoma. Gastroenterology 2011;140:1063-1070.

[47] Peng SY, Chen WJ, Lai PL, Jeng YM, Sheu JC, Hsu HC. High alpha-fetoprotein level correlates with high stage, early recurrence and poor prognosis of hepatocellular carcinoma: significance of hepatitis virus infection, age, p53 and beta-catenin mutations. Int J Cancer 2004;112:44-50.

[48] Hussain SP, Schwank J, Staib F, Wang XW, Harris CC. TP53 mutations and hepatocellular carcinoma: insights into the etiology and pathogenesis of liver cancer. Oncogene 2007;26:2166-2176.

[49] Huang J, Deng Q, Wang Q, Li KY, Dai JH, Li N, et al. Exome sequencing of hepatitis B virus-associated hepatocellular carcinoma. Nat Genet 2012;44: $1117-1121$.

[50] Fujimoto A, Totoki Y, Abe T, Boroevich KA, Hosoda F, Nguyen HH, et al. Wholegenome sequencing of liver cancers identifies etiological influences on mutation patterns and recurrent mutations in chromatin regulators. Nat Genet 2012;44:760-764.

[51] Budhu A, Forgues M, Ye QH, Jia HL, He P, Zanetti KA, et al. Prediction of venous metastases, recurrence, and prognosis in hepatocellular carcinoma based on a unique immune response signature of the liver microenvironment. Cancer Cell 2006;10:99-111.

[52] Hoshida Y, Villanueva A, Kobayashi M, Peix J, Chiang DY, Camargo A, et al. Gene expression in fixed tissues and outcome in hepatocellular carcinoma. N Engl J Med 2008;359:1995-2004.

[53] Okamoto M, Utsunomiya T, Wakiyama S, Hashimoto M, Fukuzawa K, Ezaki T, et al. Specific gene-expression profiles of noncancerous liver tissue predict the risk for multicentric occurrence of hepatocellular carcinoma in hepatitis $C$ virus-positive patients. Ann Surg Oncol 2006;13:947-954.

[54] Hoshida Y, Villanueva A, Sangiovanni A, Sole M, Hur C, Andersson KL, et al. Prognostic gene-expression signature for patients with hepatitis C-related early-stage cirrhosis. Gastroenterology 2013;144:1024-1030.

[55] Hoshida Y, Moeini A, Alsinet C, Kojima K, Villanueva A. Gene signatures in the management of hepatocellular carcinoma. Semin Oncol 2012;39:473-485.

[56] Schirmacher P, Bedossa P, Roskams T, Tiniakos DG, Brunt EM, Zucman-Rossi J, et al. Fighting the bushfire in HCC trials. J hepatol 2011;55:276-277.

[57] Kudo M. Adjuvant therapy after curative treatment for hepatocellular carcinoma. Oncology 2011;81 (Suppl 1):50-55.

[58] Trinchet JC, Chaffaut C, Bourcier V, Degos F, Henrion J, Fontaine H, et al. Ultrasonographic surveillance of hepatocellular carcinoma in cirrhosis: a randomized trial comparing 3- and 6-month periodicities. Hepatology 2011; 54:1987-1997.

[59] Cardoso F, Van't Veer L, Rutgers E, Loi S, Mook S, Piccart-Gebhart MJ. Clinical application of the 70-gene profile: the MINDACT trial. J Clin Oncol 2008;26: 729-735.

[60] Albain KS, Barlow WE, Shak S, Hortobagyi GN, Livingston RB, Yeh IT, et al. Prognostic and predictive value of the 21 -gene recurrence score assay in postmenopausal women with node-positive, oestrogen-receptor-positive breast cancer on chemotherapy: a retrospective analysis of a randomised trial. Lancet Oncol 2010;11:55-65.

[61] Clavien PA, Lesurtel M, Bossuyt PM, Gores G], Langer B, Perrier A. Recommendations for liver transplantation for hepatocellular carcinoma: an international consensus conference report. Lancet Oncol 2012;13:e11-e22.

[62] Schwartz M, Dvorchik I, Roayaie S, Fiel MI, Finkelstein S, Marsh JW, et al. Liver transplantation for hepatocellular carcinoma: extension of indications based on molecular markers. J Hepatol 2008;49:581-588.

[63] Dvorchik I, Schwartz M, Fiel MI, Finkelstein SD, Marsh JW. Fractional allelic imbalance could allow for the development of an equitable transplant selection policy for patients with hepatocellular carcinoma. Liver Transpl $2008 ; 14: 443-450$. 